This article has been published in Metallurgical and Materials Transactions A. The final publication is available at Springer via https://doi.org/10.1007/s11661-017-4097-7

\title{
A combined numerical-experimental approach to quantify the thermal contraction of A356 during solidification
}

\author{
J.P. Macht ${ }^{1}$, D.M. Maijer ${ }^{1}$, A.B. Phillion ${ }^{2}$ \\ Corresponding Author: andre.phillion@mcmaster.ca \\ ${ }^{1}$ Department of Materials Engineering, The University of British Columbia, Vancouver, Canada, \\ V6T $1 Z 4$ \\ ${ }^{2}$ Department of Materials Science and Engineering, McMaster University, Hamilton, Canada, \\ L8S 4L7
}

\begin{abstract}
A process for generating thermal contraction coefficients for use in the solidification modelling of aluminum castings is presented. Sequentially-coupled thermal stress modelling is used in conjunction with experimentation to empirically generate the thermal contraction coefficients for a strontium modified A356 alloy. The impact of cooling curve analysis on the modelling procedure is studied. Model results are in good agreement with experimental findings, indicating a sound methodology for quantifying the thermal contraction. The technique can be applied to other commercially relevant aluminum alloys, increasing the utility of solidification modelling in the casting industry.
\end{abstract}

\section{Introduction}

The coefficient of thermal expansion strongly influences the geometry and residual stress in as-cast aluminum components, and must be considered as part of the design process. In fully solid materials, temperature changes manifest as variations in material density, resulting in thermal strains that can lead to thermal stresses when a component is constrained. These thermal strains are commonly measured via dilatometry. In semi-solid materials, the situation is more complex. On one hand, the transition from liquid to solid induces a significant density difference, resulting in large solidification contraction as compared to the expected thermal contraction of the solid phases. On the other hand, liquid feeding, shrinkage porosity, and plastic deformation of the solid phase partially negate some of the effects resulting from solidification contraction. This results in complex, large (relative to single-phase thermal expansion/contraction), and nonlinear contraction behavior that occurs throughout the solidification regime [1]. Note that in this 
37 article we define the term solidification contraction to represent the thermal contraction

38 occurring within the semi-solid temperature range. Further, we define the term rigidity point as

39 the fraction solid at which a continuous dendritic network is formed, and the material starts to

40 develop strength. When the fraction solid is above the rigidity point, the solidification

41 contraction of the solid at the microstructural level can cause geometric distortions, and residual

42 stresses at the component level. However, when the fraction solid is below the rigidity point, the

43 solidification contraction is mostly compensated by fluid flow.

44 Eskin et al. developed an experimental technique based on an idea proposed by Novikov

45 [2] to measure linear solidification contraction [3]. The apparatus consists of a T-shaped graphite 46 mould that is placed on a water-cooled copper chill, and a moving wall at the base that is connected

47 to a linear-voltage displacement sensor (LVDT). The T-shaped geometry ensures that the 48 horizontal arms restrain the rest of the casting. The displacement of the moving wall as a function 49 of temperature within this casting is measured during each experiment. Eskin et al. used the LVDT 50 data to calculate a temperature-dependent thermal contraction coefficient (TCC) at high sub51 solidus temperatures,

$52 \quad T C C=\frac{\Delta L / L_{\text {gauge }}}{\Delta T}$

53 where $\Delta T$ represents the change in temperature for a given time increment at a position in the

54 casting near the moving wall, $\Delta L$ represents the change in position of the displacement sensor for 55 the same time increment, and $L_{\text {gauge }}$ is the initial length of the sample. This apparatus was used to 56 test a number of wrought aluminum alloys as well as steels, demonstrating a positive correlation

57 between the magnitude of solidification contraction and the occurrence of hot tearing.

58 Finite Element Analysis (FEA) is routinely used to model industrial casting processes.

59 Heat transfer, fluid flow, and stresses can all be simulated to understand material behavior and

60 defect formation. To accurately model stress development, knowledge of the thermal contraction

61 behavior occurring during solidification is key. However, because of its highly non-linear

62 behavior, solidification contraction is challenging to quantify consistently and its study requires a

63 multi-faceted approach. The approach used by Eskin to measure linear solidification contraction

$64[3]$ is useful for obtaining the required experimental data. However, because of strong thermal 65 gradients within a casting, Eq. 1 provides only an estimate of the solidification contraction, and 66 not quantified solidification contraction coefficients that can be used for modelling purposes. 
In this research, a combined experimental/numerical method is presented to quantify the thermal strains and the corresponding solidification contraction coefficients experienced by aluminum alloys. The Al-Si casting alloy A356 is used as the exemplar system. First, small ingots are cast following a method similar to Eskin's approach to measure solidification contraction. Second, thermal-stress numerical modeling is used to quantify the heat transfer and thermal contraction behavior during solidification; the input solidification contraction coefficients are adjusted in successive model iterations until the overall linear contraction predicted by the model matches the experimentally-measured linear contraction. This new coupled methodology provides insight into high-temperature solidification contraction of metals, and can be easily applied to other systems in order to quantify the solidification contraction coefficients required for predicting hot tearing in macro-scale casting models.

\section{Experimental}

Materials: The material used in this study was strontium-modified A356 (Al - $7 \mathrm{wt} \% \mathrm{Si}, 0.3 \mathrm{wt} \%$ $\mathrm{Mg}, 0.003 \mathrm{wt} \% \mathrm{Na}, 0.008 \mathrm{wt} \% \mathrm{Sr}$ ). This Al-Si foundry alloy is prevalent in the automotive industry due to its good castability and good mechanical properties that can be obtained through heat treatment [4]. Strontium modification is employed to alter the morphology of the Si precipitate from a blocky structure to an interconnected fibrous phase [5]. Apparatus Design: The experimental setup used to measure the linear contraction is based on the T-shape design of Eskin et al. mentioned in the Introduction. An image of the experimental apparatus, along with the dimensions of the mold and an image of the sample itself are given in Figure 1. Note that while Eskin [3] placed the water-cooled copper chill below the graphite mold, it is placed at the head of the T-shape in this work.

90 The goal of these experiments is to measure the horizontal linear contraction. Moving the water91 cooled copper chill to the head of the T-shape causes directional solidification in the horizontal 92 direction, which then enables the horizontal contraction to be measured directly. Specifically, the 93 horizontal linear contraction is measured by recording the displacement of a clearance-fit Invar 94 rod that is embedded into the ingot through the moving wall at the base of the leg of the T-shape. 95 The head of the T-shape provides a restraint that the leg of the T-shape contracts against. During 96 casting, the melt solidifies and then contracts around one end of the rod while displacement is 97 recorded using an LVDT (HR Series General Purpose LVDT configured with an ATA-2001 
Analog LVDT/RVDT Signal Conditioner fabricated by Measurement Specialties Ltd.) mounted

99 to the other end of the rod. It is important to note that contraction will also occur in the vertical

100 direction of the casting because of heat transfer between the casting and the environment.

101 Maintaining a truly unidirectional thermal gradient in the mold cavity and consequently

102 unidirectional thermal contraction for the duration of the experiment is a challenging heat transfer

103 requirement that is not met with this experimental methodology.

104 Understanding the temperature profile of the castings is essential to understanding the 105 manner in which thermal stresses ultimately develop. For the temperature measurements, three 106 type-K thermocouples are placed near the head (closest to the chill), center, and base (closest to 107 the moving wall) of the T shape. These thermocouples were located at a height of $\sim 12.5 \mathrm{~mm}$ below 108 the open top of the mold.

109 Experimental Procedure: The experimental procedure consisted of first melting the A356 in a 110 holding furnace, then pouring the melt into the graphite T-shaped mold at a casting temperature of $111888 \mathrm{~K}\left(615^{\circ} \mathrm{C}\right)$, and finally recording the displacement of the moving wall and the temperature 112 evolution within the casting during solidification at a rate of $5 \mathrm{~Hz}$ until the center thermocouple 113 reached $723 \mathrm{~K}\left(450^{\circ} \mathrm{C}\right)$. Cold water was passed through the copper chill continuously at a flow 114 rate of $20 \mathrm{~L} / \mathrm{min}$. In total, three experiments (Trials $\mathrm{A}, \mathrm{B}$, and $\mathrm{C}$ ) were carried out using identical 115 processing conditions. The average cooling rates measured during solidification for each 116 experiment are listed in Table 1.

\section{Numerical}

118 Model Formulation: A 3D sequentially coupled thermal - stress model was developed within the 119 Abaqus FEA software to simulate the evolution of temperature and stress/strain fields within the 120 T-shaped casting during solidification and cooling to $723 \mathrm{~K}\left(450^{\circ} \mathrm{C}\right)$. This model was applied to 121 each of the three experiments. The term sequentially coupled implies that first the thermal field is 122 calculated without consideration of the effects of stress, and then second the elastic stress analysis 123 is performed using the nodal temperatures from the thermal analysis as a predefined field. The 124 advantages of sequential coupling are twofold: (1) computational time is reduced and (2) multiple 125 stress analyses with different material properties can be simulated for a given thermal analysis. 126 The use of sequential coupling allowed for the decoupling of the objectives sought within each 127 stage of the modelling procedure - tuning of the heat transfer coefficients in the thermal model 128 and then quantifying the solidification contraction coefficients in the stress model. 
130 Geometry: The thermal model geometry consisted of one half of the T-shaped casting, as centerline

131 symmetry was assumed (Figure 2a), as well as one-half of the graphite mold (Figure 2b).

132 Initial Conditions: The nodal temperatures of the casting and die were initially set to $888 \mathrm{~K}\left(615^{\circ} \mathrm{C}\right)$ 133 and $298 \mathrm{~K}\left(25^{\circ} \mathrm{C}\right)$, respectively.

134 Boundary Conditions: To extract heat, a series of heat transfer coefficient boundary conditions 135 were applied between the casting and/or mold and the surrounding environment. The values used 136 to represent heat loss to the environment are given in Table 2, and were inferred from previous 137 work [6]. These values were used for the thermal simulations of all three casting trials. The heat 138 transfer across the interface between the casting and mold was modelled via a thermal contact 139 conductance coefficient using the GAPCON user-written subroutine within the Abaqus FEA 140 software. The temperature-dependent coefficients, given in Table 3, were tuned such that the 141 predicted temperatures matched the experimental cooling curve collected at the center of the 142 casting, TC2. This tuning process was performed on each thermal simulation in order to account 143 for the observed differences in temperature evolution between the three casting trials. As can be 144 seen in Table 3, the coefficients are slightly different between Trials A, B, and C. The fraction 145 solid development and its inherent relationship to latent heat release was also incorporated into the 146 model tuning process using the equation-based Newtonian (EBN) cooling curve analysis [7] 147 developed by Gibbs and Mendez (the reason for and results of which are discussed later).

148 Material Properties: The thermo-physical (density, thermal conductivity, heat capacity and total 149 latent heat) properties of A356 and graphite were based on values reported in the literature [4, 8]. Stress Model

151 Geometry: As friction between the mold and casting was assumed to be minimal, only one-half of 152 the T-shape casting was included in the stress model.

153 Boundary Conditions: To provide mechanical restraint, it was assumed that the two surfaces of the 154 T-shape providing the restraint remained in contact with the mold, (i.e. $u_{x}=u_{y}=u_{z}=0 \mathrm{~mm}$, where $u$ 155 is the displacement in the $x, y$, or $z$, directions) as shown in Figure 2. The base of the casting was 156 also assumed to remain in contact with the mold $\left(u_{z}=0\right)$.

157 Material Properties: The Young's modulus and Poisson's ratio of A356 and graphite were based 158 on values reported in the literature [9, 10]. Following Hao et al. [11], it was assumed that the 159 Young's modulus is only of significant magnitude at temperatures below the rigidity point, 
160 whereas above, it is only a small value. The rigidity point was assumed to occur at the temperature

161 where the non-equilibrium primary eutectic reaction begins to occur, $T_{\text {Rigid }}=568^{\circ} \mathrm{C}$. The

162 solidification contraction coefficients are the focus of this research and will be presented in the

163 next section. The UEXPAN user-written subroutine was used in Abaqus to model the relevant

164 thermal expansion behavior. The values were empirically determined via the tuning process such

165 that one set of solidification contraction coefficients was used for the thermal-stress simulations

166 of all three trials, while still producing accurate representations of the thermal contraction behavior

167 seen in each experimental trial.

168

169

170

171

172

173

\section{Results and Discussion}

The evolution in temperature measured at TC2 during each casting experiment is shown in Figure 3a. As can be seen, the metal first underwent cooling in the liquid state, followed by primary solidification, then eutectic solidification, and finally solid state cooling. All three curves appear quite similar, demonstrating the repeatability of the methodology.

The thermocouple measurements TC1, TC2, and TC3 from Trial A are shown in Figure 3b along with the corresponding results from the numerical simulations. The cooling observed at these three locations is different due to their relative distance from the copper chill. Within the thermal model outlined in Section 3, the main adjustable parameter that affects cooling of the casting is the heat transfer coefficient between the casting and the mold. Still, as can be seen in Figure $3 \mathrm{~b}$, the predicted thermal profiles at TC1 and TC3 match well, demonstrating the validity of the chosen heat transfer coefficients. Any slight time delay discrepancy on fit for any given model-experiment thermocouple pair is attributed to the slight variability in contact time between the melt and the thermocouple and/or error in knowledge of the thermocouple location.

Table 2 shows that the tuned heat transfer coefficients for Trial $\mathrm{C}$ are approximately $10 \%$ larger than for Trials $\mathrm{A}$, and $\mathrm{B}$ at temperatures where solidification is taking place. Correspondingly, Table 1 shows that the average cooling rate during solidification for Trial $\mathrm{C}$ was approximately $10 \%$ larger than for Trials A and B. We hypothesize that this difference is due to our inability to pour the liquid metal in an entirely repeatable fashion; with such a small casting, even slight variation in the initial location of melt/mold contact and trajectory of the pour has an impact on the rate of cooling, especially at high temperatures. Note however, that all three trials resulted in similar microstructure, as confirmed via measurements of secondary dendrite arm 
191 spacing (SDAS), which was found to be: $\lambda_{2, \text { Trial } A}=25.2 \mu \mathrm{m}, \lambda_{2, \text { Trial } B}=23.1 \mu \mathrm{m}$ and $\lambda_{2, \text { Trial } C}=$ $19223.2 \mu \mathrm{m}$. This small spacing is linked to the high cooling rate experienced during solidification (5$19315 \mathrm{~K} / \mathrm{s}$ ) of each casting [8].

194 Due to the small size of the casting, $\sim 200 \mathrm{~g}$, the manner in which latent heat is evolved 195 within the model plays a significant role in ensuring that the predictions match the experimental 196 data. Further, for industrial alloys with a considerable fraction of eutectic, like A356, the 197 temperature over which the non-equilibrium eutectic transformation occurs, and hence the latent 198 heat evolves, is uncertain and highly variable as it depends on cooling conditions and local 199 composition. Our initial simulations estimated the evolution in latent heat using a fraction solid / 200 temperature curve based on a relationship found in the literature [4]. However, through the iterative 201 process of tuning the heat transfer coefficients, it became clear that the thermal model could not 202 be fit to the experimental data unless an improved evolution in fraction solid with temperature 203 curve could be determined matching the experimental results.

204 To estimate the evolution of fraction solid versus temperature that occurred during the Tshaped casting experiments, the equation-based Newtonian (EBN) method of cooling curve 206 analysis [7] by Gibbs and Mendez was applied. The results, based on the temperature measurements at TC2, are shown in Figure 4 for each experiment. As can be seen, the fraction solid versus temperature curves for Trials $\mathrm{A}$ and $\mathrm{C}$ are quite similar, while the curve for Trial $\mathrm{B}$ seems shifted. It is hypothesized that perhaps the melt in Trial B was poured in such a way that a 210 coherent dendritic structure formed almost immediately along the bottom of the mold, accelerating 211 the evolution in fraction solid. However, the thermal curves for Trials A and B were quite similar, 212 and the transformation temperatures for all three trials all seem to agree with each other despite 213 the shift in Trial B fraction solid. The observed delayed onset of the solidus temperature as 214 compared to the phase diagram is in agreement with phenomena reported by Thompson [4]. The 215 formation of $\mathrm{Mg}_{2} \mathrm{Si}$ (not typically seen in near-equilibrium cooling conditions [12]) also appeared 216 at temperatures consistent with those reported by Thompson at high cooling rates. The data from 217 each curve was used for the individual casting simulations, to capture the observed variations in 218 latent heat evolution. This cooling curve analysis is a key feature of the combined 219 numerical/experimental method for determining solidification contraction coefficients in A356.

220 The measured displacements of the moving wall for all three trials are shown in Figure 5a. 221 These values are negative because the contraction of the casting causes the wall to move into the 
222 mold. As can be seen, all three trials produced very similar results, demonstrating the robustness 223 of the T-shaped casting mold for measuring solidification contractions. The values of the TCC 224 parameter based on the experimental data, calculated using Eq. (1), are shown in Figure $5 \mathrm{~b}$ as a 225 function of temperature at TC2. This data provides significant insight into solidification 226 contraction. First, it would appear that contraction initiates at a temperature where the non227 equilibrium primary eutectic reaction begins to occur, at $841 \mathrm{~K}\left(568^{\circ} \mathrm{C}\right)$ as was assumed in Section 228 2. Then, the rate of contraction varies significantly as the casting cools, increasing and decreasing 229 at various temperatures. While some of the observed features will be linked to the fact that there 230 is a temperature gradient along the longitudinal direction, the temperature corresponding to 231 changes in behavior generally matches with the initiation of the primary eutectic reaction, the 232 formation of $\mathrm{Mg}_{2} \mathrm{Si}$ and the depressed solidus temperature. Note that the TCC parameter values 233 reported in Figure $5 \mathrm{~b}$ are not the same as a thermal strain coefficients, nor the coefficient of thermal 234 contraction/expansion. The TCC parameter represents a change in casting length relative to the 235 gauge length of the sample as a function of temperature, as noted in Equation 1. Thus, the TCC 236 parameter is a measurement of the integrated dimensional change of the casting relative to a 237 measured temperature at some point (in this case, TC2). This is different than a strain occurring at 238 an infinitesimal point in the casting due to a thermal change at that same point, which is what the 239 thermal strain coefficient represents. The values are in the same order of magnitude and are 240 equivalent in unit due to the similar characteristics they represent. The value of the thermal strain 241 coefficient for the high temperature, solid phase $\alpha$ for LM25 (Al-7Si-0.2Cu, $\alpha=2.6 \cdot 10^{-5} \mathrm{~K}^{-1}$ ), reported by Mills [8] has been included in Figure $5 \mathrm{~b}$ for comparison.

243 Using the experimentally-measured displacement data and corresponding TCC parameter 244 values, an iterative process was applied to determine the thermal strain coefficients required to 245 accurately model the displacement of the moving wall. Initially, the entries in the UEXPAN user246 written subroutine of the Abaqus FE solver matched the TCC parameter data. The values were 247 then modified to improve the fit of the predictions to the experimental displacement curves 248 presented in Figure 5a. The final thermal strain coefficients used in all three stress simulations are 249 reported in Table 4 as a function of temperature.

250 The model-predicted displacement of the moving wall as a function of time is also shown 251 in Figure 5a. As can be seen, through parameter tuning, a very good match is achieved for Trials 252 A and C. The fit for Trial B is weaker, especially at early times. This is related to the difference in 
253 fraction solid evolution as observed in Figure 4. The corresponding predicted TCC parameter

254 values are given in Figure 5b. Here, there is generally good agreement between the experimental 255 and simulated results, although some of the fine features are not captured. Most importantly, the 256 predicted TCC parameter values vary significantly as the casting cools in the same manner as the 257 measured TCC parameter values, both increasing and decreasing at similar temperatures.

258 The displacement measurements and predictions shown in Figure 5a can also be compared

259 to the known solidification shrinkage value for the A356 alloy, 4.1\% based on the density 260 comparison $\left(\rho_{\text {liq }}=2420 \mathrm{~kg} / \mathrm{m}^{3}\right.$ and $\rho_{\text {sol }}=2550 \mathrm{~kg} / \mathrm{m}^{3}$ [13]). If the assumption is made that the T-

261 shaped casting only contracts on the open top face and the moving wall, the overall volumetric 262 changes between the liquid and solid states using the displacement magnitudes recorded in Figure $2635 \mathrm{a}$ is calculated to be approx. $\sim 1.15 \%$. The significant difference between this value and the known 264 solidification shrinkage is thought to manifest through the slight gaps formed at casting mold 265 interfaces and on the corners of the mold.

\section{Conclusions}

In this study, the solidification contraction of an aluminum A356 alloy has been measured using 269 a combined numerical/experimental approach. This temperature-dependent material property, 270 knowledge of which is critical for improving casting quality, is extracted by tuning the thermal 271 strain coefficients within a thermal/stress simulation based on their similarity to the measured 272 evolution in casting contraction. Key to this analysis is (1) the use of a T-shaped mold which 273 ensures oriented contraction, and (2) cooling curve analysis to accurately represent the release of 274 latent heat within the mathematical simulation. The measured thermal contraction coefficients 275 can be used as part of process models to improve the predictions of thermal contraction during 276 aluminum shape casting processes.

\section{Acknowledgements}

278 The authors gratefully acknowledge the assistance of C. Reilly, J. Kantharaju and S. Bhatia for 279 their help in conducting the casting trials and developing the model, as well as E. Smith for 280 constructive feedback on the manuscript text. This research was funded by the Natural Sciences 281 and Engineering Research Council of Canada.

\section{References}

283 [1] J. Campbell: Castings, Butterworth-Heiniemann, Oxford, 1991. 
[2] I.I. Novikov: Goryachelomkost tsvetnykh metallov i splavov (Hot Shortness

[3] D.G. Eskin, Suyinto, J.F. Mooney, L. Katgerman: Metall Mater Trans A, 2004, vol. 35A, pp. 1325-1335.

[4] S. Thompson. Effect of cooling Rate on Solidification Characteristics of Aluminum Alloys A356 and AA5182. MASc Thesis. The University of British Columbia, Vancouver, Canada, 2003.

[5] O. Elsebaie, A.M. Samuel, F.H. Samuel: J Mater Sci, 2011, vol. 46, pp. 3027-3045.

[6] B. Zhang, D.M. Maijer, S.L. Cockcroft: Mater Sci Eng A, 2007, vol. 464, pp. 295-305.

[7] J.W. Gibbs, P.F. Mendez: Scripta Mater, 2008, vol. 58, pp. 699-702.

[8] K.C. Mills: Recommended Values of Thermophysical Properties for Selected Commercial Alloys, Woodhead Publishing Ltd, Cambridge, England, 2002, pp. 43-49.

[9] H.J. Frost, M.F. Ashby. Deformation-Mechanism Maps: The Plasticity and Creep of Metals and Ceramics, Pergamon Press, Oxford, 1982.

[10] M.J Roy, D.M. Maijer: Light Metals 2012, ed. C.E. Suarez, John Wiley \& Sons Inc., Hoboken, NJ, USA. 2012, pp. 377-382.

[11] H. Hao, D.M. Maijer, M.A. Wells, A. Phillion, S.L. Cockcroft: Metall Mater Trans A, 2010, vol. 41A, pp. 2067-2077.

[12] H.B. Dong, M.R.M. Shin, E.C. Kurum, H. Cama, J.D. Hunt: Metall Mater Trans A, 2003, vol. 34A, pp. 441-447.

[13] S. Thermalingam, C.H. Caceres, J.A. Taylor: Metall Mater Trans A, 2013, vol. 44A, pp. 4071-4080. 

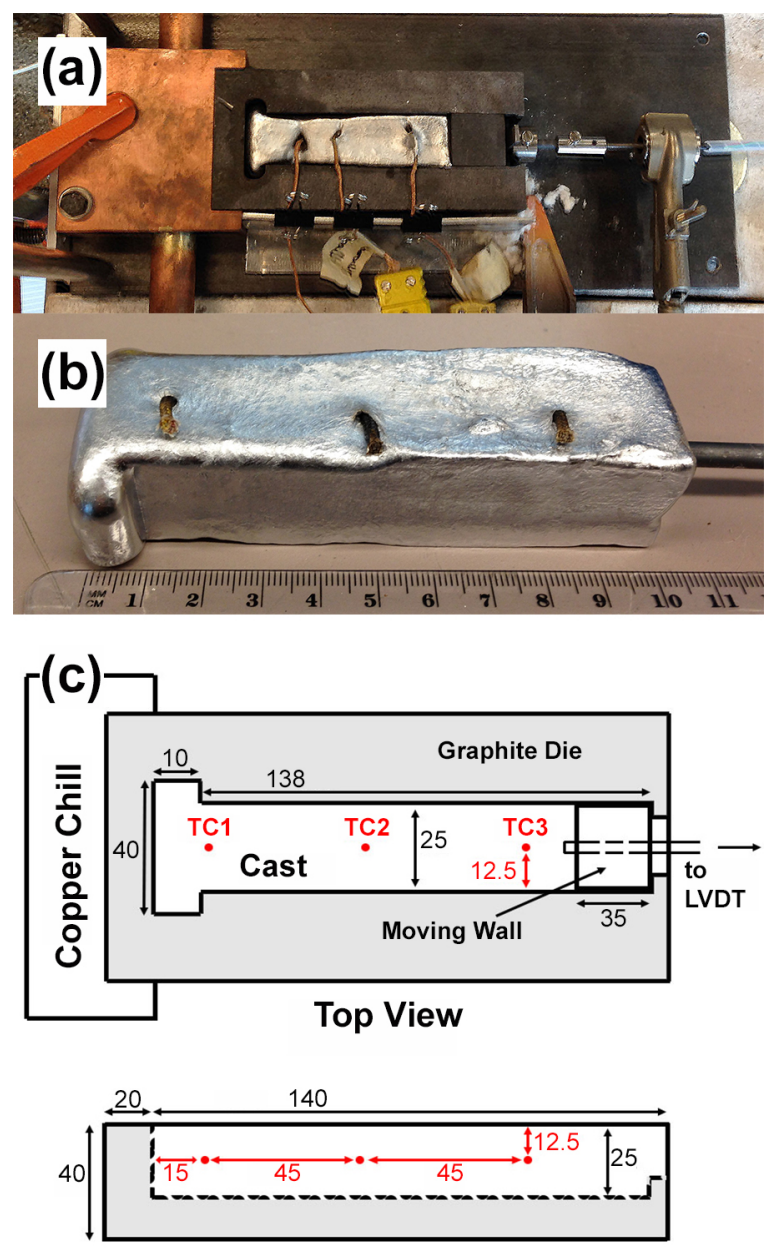

Side View

Figure 1 (a) Experimental setup of graphite mold, copper chill, LDVT and T-shaped casting, (b) and thermocouple locations. 

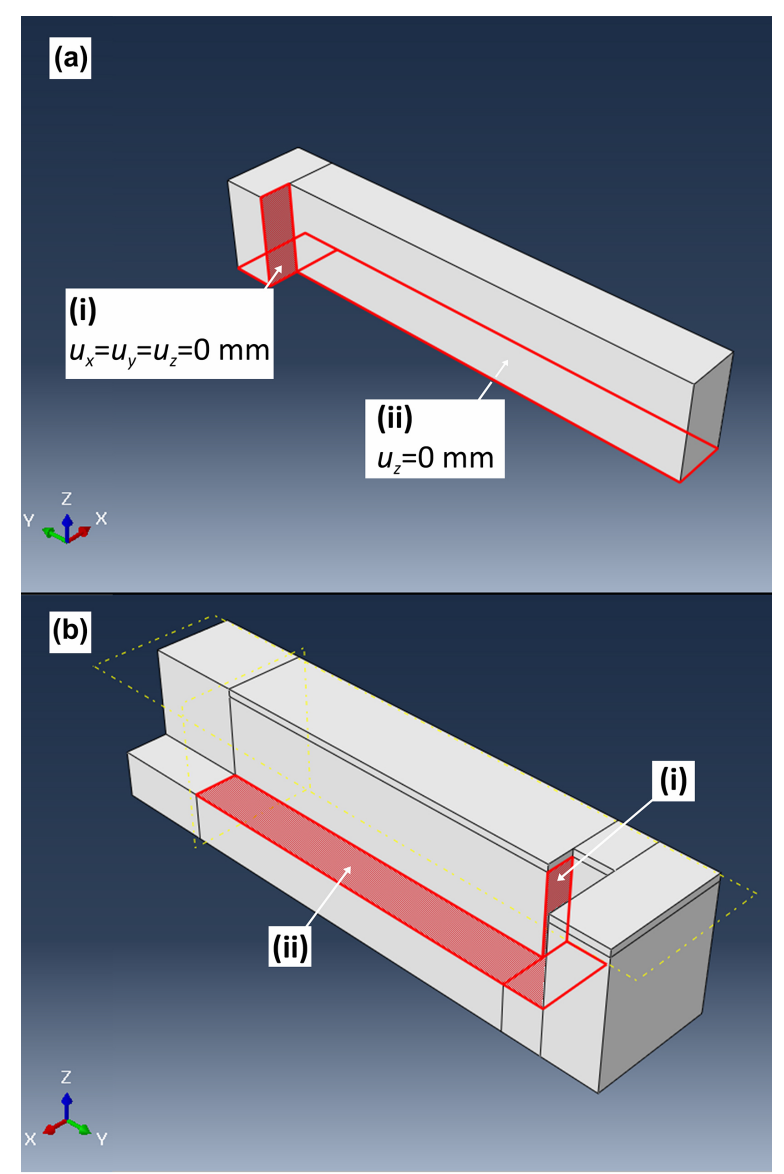

Figure 2 (a) Model of cast ingot showing the mechanical constraints; (b) Model of the graphite die, rotated 180 degrees about the z-axis, with corresponding constraint surfaces labelled.
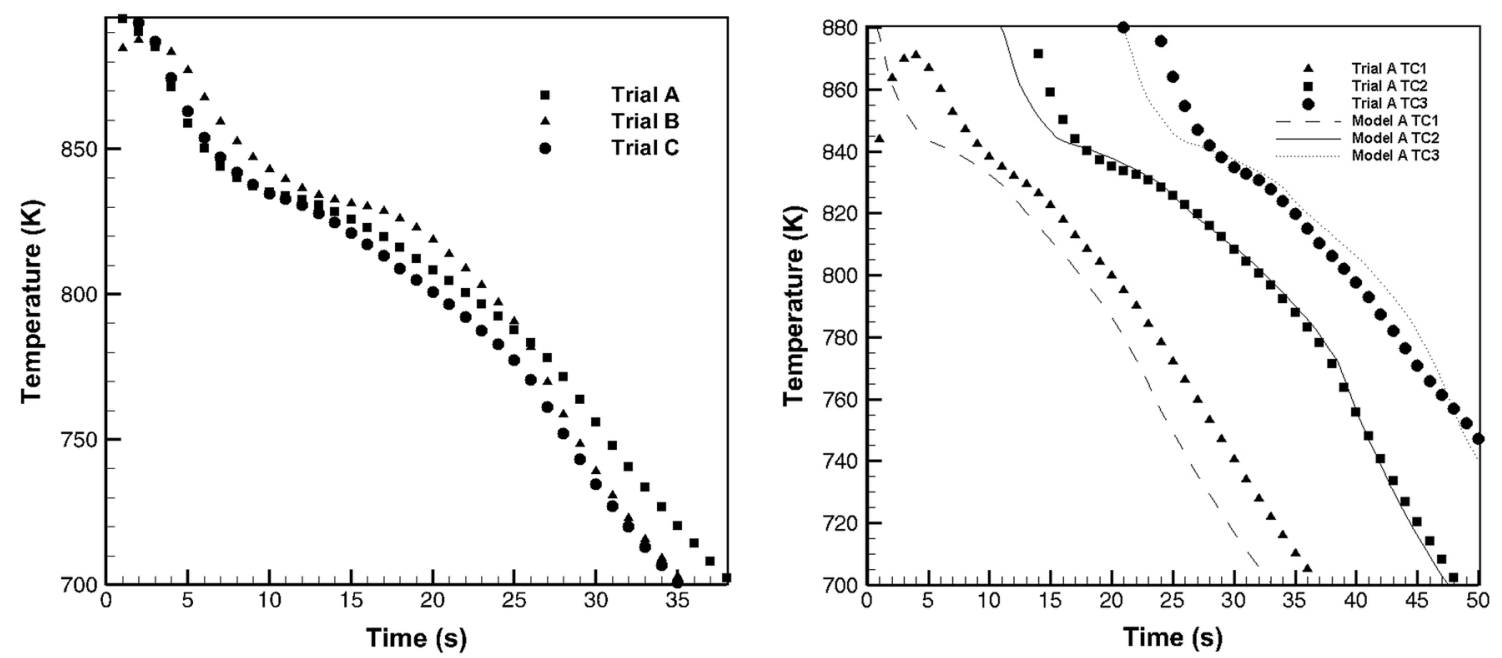

Figure 3 (a) Experimentally-measured time-temperature curves at TC2 for Trials A, B, and C; shifted by 10 s to more clearly show the comparison. 


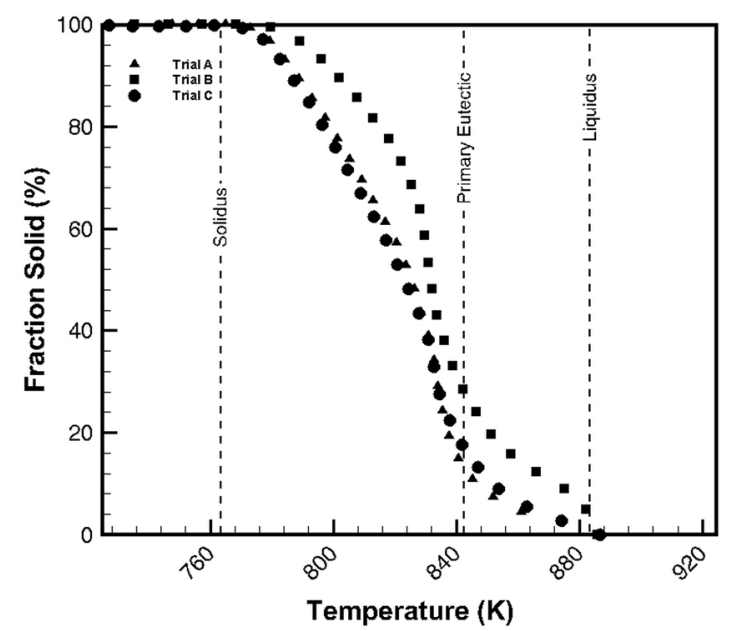

$332 \quad$ Figure 4 Evolution in fraction solid with temperature as modelled by the EBN method.

(a)

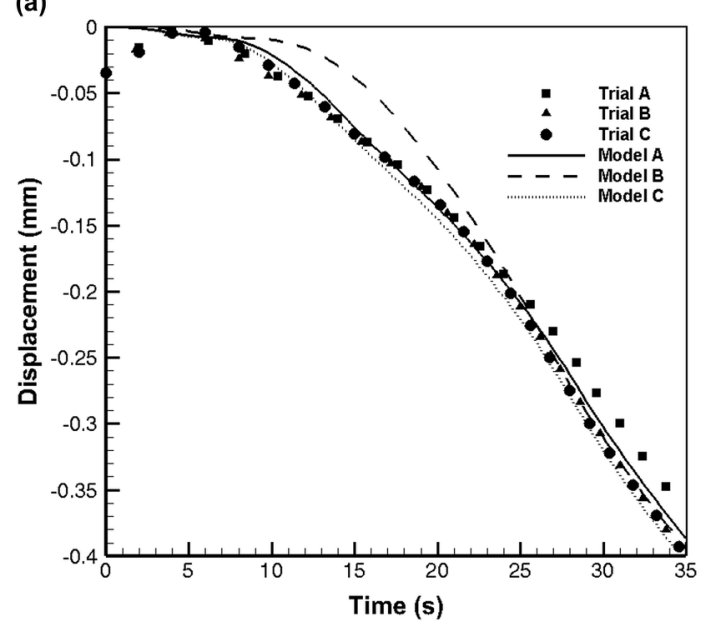

(b)

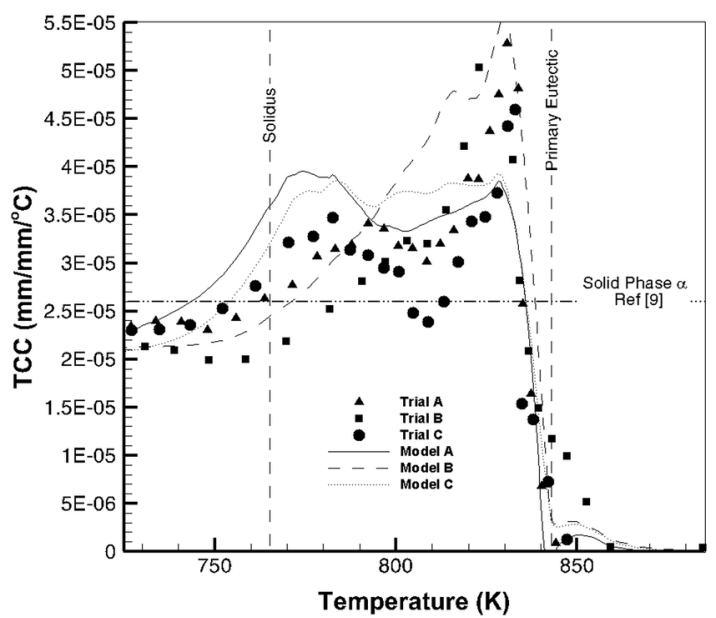

Figure 5 (a) Displacement during contraction (b) TCC parameter as calculated using the central thermocouple, TC2. 
Table 1: Average cooling rates during solidification observed in experiments

\begin{tabular}{cccc}
\hline \multirow{2}{*}{ Temp } & \multicolumn{3}{c}{$(\mathbf{K} / \mathbf{s})$} \\
\cline { 2 - 4 } & $\begin{array}{c}\text { Trial } \\
\mathbf{A}\end{array}$ & Trial B & Trial C \\
\hline $\begin{array}{c}883-763 \mathrm{~K} \\
\left(613-490^{\circ} \mathrm{C}\right)\end{array}$ & 4.8 & 4.8 & 5.3 \\
\hline
\end{tabular}

340

341

342

343

\begin{tabular}{cccc}
\hline Table 3: Heat transfer coefficients for the cast & \multicolumn{3}{c}{ HTC $\left(\mathbf{W} / \mathbf{m}^{2} \cdot \mathbf{K}\right)$} \\
\cline { 2 - 4 } Temp & Trial & Trial B & Trial C \\
\hline & A & & 2300 \\
$923 \mathrm{~K}$ & 2300 & 2300 & \\
$\left(650{ }^{\circ} \mathrm{C}\right)$ & & & \\
$873-778 \mathrm{~K}$ & 965 & 940 & 1045 \\
$\left(600-505{ }^{\circ} \mathrm{C}\right)$ & & & \\
$763-713 \mathrm{~K}$ & 400 & 420 & 450 \\
$\left(490-440^{\circ} \mathrm{C}\right)$ & & & \\
$698 \mathrm{~K}$ & 350 & 350 & 350 \\
$\left(425{ }^{\circ} \mathrm{C}\right)$ & & & \\
\hline
\end{tabular}

Table 2: Heat Transfer Coefficients used in the Thermal Model of the T-shaped casting

\begin{tabular}{cc}
\hline Interface & HTC $\left(\mathbf{W} / \mathbf{m}^{\mathbf{2}} \mathbf{K}\right)$ \\
\hline Casting/Air \& Mould /Air & 50 \\
Mould/Support Table & 500 \\
Mould/Copper chill & 3000 \\
Casting/Moving Wall & 600 \\
Casting/Mould & Discussed below \\
\hline
\end{tabular}

Table 3: Heat transfer coefficients for the casting / mould interface.

345 Table 4: Thermal strain coefficients (including solidification contraction coefficients) used in the 346 modelling of thermal contraction during solidification of Sr modified A356.

\begin{tabular}{cc}
\hline Temp & $(\mathbf{m m} / \mathbf{m m} \cdot \mathbf{K})$ \\
\hline $841 \mathrm{~K}$ & 0.1 \\
$\left(568^{\circ} \mathrm{C}\right)$ & \\
$823 \mathrm{~K}$ & 4.0 \\
$\left(550^{\circ} \mathrm{C}\right)$ & \\
$806 \mathrm{~K}$ & 2.7 \\
$\left(533^{\circ} \mathrm{C}\right)$ & \\
$763 \mathrm{~K}$ & 3.2 \\
$\left(490^{\circ} \mathrm{C}\right)$ & \\
$753 \mathrm{~K}$ & 2.5 \\
$\left(480^{\circ} \mathrm{C}\right)$ & \\
\hline
\end{tabular}

